\title{
Currents Deiven by Electron Cyclotron Waves
}

\section{by}

Charles E. F. Rarney and Nathantel J. Fisch

Plasma Fhysics Iaboratory, Princeton Ditversity

Princeton, New Jersey 08544 USA

Abstract

Certain aspects of the generation of steady-state currents by electron cyclotron waves are exploted. A numerical solntion of the Fokk-r-planck equation is used to verify the theory of $315 \mathrm{sh}$ and bozer and to extend their resuit:s into the nonlisear regtme. Ralativistic effects on the sucrent generated are discussed, Applicarions to stealy-state tokandk reactors ars considered. 


\section{INTRODUCTION}

The generation of electric currents in plasma by means of electron cyclotron wave absorption appearg to be one of the more promising schemes of providing a steady-state toroidal current in a tokamak.' These waves can be employed to generate toroidal current merely by heating selected electrons and, Interestingly, without directly injecting substantial toroidal momentum into these electrons. The wave launching structures are advantagsously simple; because the wave noed not have high parallel to $B$, the dc magnetic field) monentun content, its parzllel phase velocity can be superluminous and, acrordingly, no slow wave structure is necessary. Moreover, the utilization of the high frequency range t the wave frequency, is, is contparable to $\Omega_{e}$, the electron cyclotron frequencyl Implies that the wave power density is also high. It follows that free space waves of high power density may be injected into the plasma through conveniently small waveguide apertures in order to drive the toroidal current.

The main problem in generating current by this means is the power requirement, both in terms of the magnitude of the recycled power in a tokamak reactor as well as the capital costs of the equipment. Efficient cw power sources for this range of frequencles are yet to be develofed. Agguming that thege sourcas can be developed, the current must still be generated with minlmal power dissipation for the scherde to be economically feasible in a fuston reactor. This minimization requires the absorption of the wave by only the fastest electrons, which are the most collisionless and hence retain their directed current longest. In this respect, this scheme is similar to the alternative technique of current generation by lower hybrid waves. 2 which also explotts, among other things, the relative infrequency with which the superthermal electrons experience collisions. 
The present scheme, however, may allow the wave to resonate even with relativistic electrons ${ }^{3}$ whereas the lower hybrid waves are constrained by an accessibility condition that, depending on the plasma $\beta$ and temperature, allows Iesonance only with somewhat slower electrons.

It is an object of the present paper to analyze, both analytically and sumerically, the raechanisms by which the absorption of electron cyclotron waves leads to the production of curfent. The paper is organlzed as follows: In section II we consider analytically the wave absorption fron the standpoint of linear theory in a slab model low density plasma. This simplified analysis nonetheless indicates the most promising injection angle of the wave into the tokamak and reagonably estinates the speed of the electrons thai absorb the wave. In section III we check numerically the formula given in Ref. I for $J / P d$, the current generated per power dissipated, and we find close verification of the theory. We then turn to other effects that are likely to enter the problem in important parameter regimes. In section $I V$ we consider nonlinear effects, $i$.e., the effect that Einite or even large wave power has on the amount of current generated and the wave damping rate. In section $v$ we assess the inplications of reiativistic effects ${ }^{3}$ that become pertinent in reactor grade plasmas. In Section VI we present a summary of our findings.

Throughout our discussion we shali be comparing our observations with analytical and numericas treatments of the closely related and more familiar problem of current generation by lower hybrid wavss 2,4 where the waveparticle interaction takes place at the Landau resonance, We conclude the present section with an important distinction between the two mechanisms. The resonance condition for electrons to exchange energy with the waves is 
$w-k_{1} v_{n}=n \Omega_{e}(s)$,

where $k$ is the wave parallel wavenumber, $v_{\text {i }}$ is the electron parailel yelocity, s measures distance in the direction of the tokamak major radius and for lower hybrid waves $\pi=0$, while for electron cyclotion waves $n= \pm 1$. It may he seen that, neglecting the polotaal magnetic field and toroidal curvature cffects, electrong with the same $y$ absorb the lower hybrid wave. Neac the plasma center, the plasma is hotter and denser than near the plasma periphery, so the absorption can be concentrated there as there are more electrons there to absorb the wave. This situation is depicted in FIg. 1(a), where a spectrum of waves with purposefully high parallel phase velocity is ut1lizel to avoid power absorption near the cool and underdense periphery.

In contirst, as electron cyclotron waves propagate into regions of different magnetic field, they not only resonate with mote plectrons, but they resonate with electrons of different parallel velocity. This is depicted in Fig. 1(b), which Indicates the phenomenon in the case of the extraordinary wave, which is Iaunched from the high-field s-de of the tokamak, where there are no regonant electrons. At some interlox point, however, there may be a large number of resonant electrors. Just as for the Iower-hybrid wave, as the wave propagates Inward, more and more electrons besome resonant. However, in contrast to the case of the lower-hybrid wave. the electrons becoming resonant are those that are slower and slower as the wave nears the resonant surface, $w=\Omega_{e}$ The slower electrons are less efficient to heat for generating currents so that it becomes critical that the electron cyclotron wave damp completely before v becomes too small. 


\section{WAVE ABSORPTION}

The effect of electron cyclotron resonance heating is to increase primarily the perpendicular velocity of the resonant electrons. The velocity increase lies in this direction because the waves have very Itttle parallel monentum content compared to energy content, so that when the wave is absorbed by an electron, the elect:on' anergy increages, but, by momentum conservation, its parallel momentum barely increases. That the waves themselves have little paraliel momentum to impart to the electrons is a consequence of their superluminous parallel phase velocity. The energy in a wave is proportional to $\omega$, whtle tts momentum is proportional to $k$. Since $\omega / k$, , the waves possess relatively little momentum.

Neglecting then the sinall parallel monentum of the wave (which vanishes in the limit $\left.w / k_{0}+\infty\right)$, we view the wave-partlist lit.prtistion as a diffusive provess in velocity space where

$$
\frac{\partial E}{\partial t}=\frac{1}{v_{\perp}} \frac{\partial}{\partial v_{\perp}} v_{\perp} D_{r f} \frac{\partial}{\partial v_{\perp}} f \text {, }
$$

where $f$ is the electron velocity distribution and $D_{r f}$ is the wave diffusion coefficient which may be written heuristically as $D_{r f}=\left\langle\Delta v_{1}^{2}\right\rangle / \Delta t$, where $\Delta V$ is the characteristic velocity change in an autncorrelation time $\Delta t$. The waves accelerate electrons through a perpendicular electric field, $E_{1^{\prime}}$ For extraorilndry waves we may write

$$
\Delta v_{\perp}=\frac{e}{m} E_{\perp} \Delta t
$$

where $\mathrm{e} / \mathrm{m}$ is the electron charge $1: 13$ ind:3s ratio. The correlation time of the waves is given approximately by 


$$
\Delta t=\pi / \Delta k_{1} \nabla_{1}
$$

where wave chosen the constant $\pi$ so that

$$
D_{r f}=\frac{\Delta v^{2}}{\Delta t}=\left(\frac{e E_{\perp}}{m}\right)^{2} \frac{\pi}{v_{1} \Delta k_{1}} \text {, }
$$

which is fust the result derived in a more precise manner.5 The powex dissipated mey be found from Dq. (1) as

$$
P_{d}=\int \frac{1}{2} n_{0} m v_{\perp}^{2} \frac{\partial f}{\partial t} d^{3 t}=2 n_{0} m D_{I f} \int f d^{3+} \equiv 2 n_{r} D_{I f} .
$$

where we integrated twice by parts to obtain the second equality, assuming $D_{r f}$ is independent of $v_{1}$ in a range in $v_{1}$, and we define $n_{r}$ as the number of rescrant electrons. The interpretation of Eq. (5) is that the power dissipated depends directly on the number of resonant electrons. (This result is a consequence of taking $D_{r f}$ to be independent of $v_{1}{ }^{*}$ In the linear limit, the distribution function is a Maxwellian and $n_{I}$ and the damping rate are independent of $D_{r f}$. It is possible, however, that $n_{r}$ could change due to nonlinear effects. Should $n_{r}$ Increage, then the wave damping rate, with increasing $D_{r f}$, would increase rather then decrease, in contrast to the scaling in the case of lower hybrid waves. It is difficult, however, to find $n_{r}$ andytically. We do, however, explore numerically nonlinear aspects of this problem in Section IV.

We consider now the implications of the linear theory on the efficiency of driping current. Osing Eq. (4) and assuming $k_{l}>k_{\perp}$, we may write the temporal damping of extraordinary waves as 


$$
Y=\frac{\pi \omega_{p e}^{2}}{k_{n} \Delta v_{i}} \frac{n_{r}}{n_{0}}
$$

where $\omega_{p e}$ is the electron plasma frequency, and $n_{z}$ is the density of resonant electrons in a width $\Delta v_{p}$. The spatial damping of the wave is given in the limit of underdense plagma as

$$
\alpha_{s}=\frac{\gamma}{v_{g s}}=\frac{\gamma k}{c k_{s}}=\frac{\pi}{k_{s}^{c}} \frac{\omega_{p e}^{2}}{\Delta v_{1}} \frac{n_{I}}{n_{0}} .
$$

where subscript $s$ denotes the direction that is also parallel to $R$, where $R$ is the major radius.

It is important to determine the region of veloclty space in which the largest portion of the wave energy is absorbed. The wave enters the plasma at some horizontal position $s=s_{a}$ and eventually loses its power at some position $s=s_{b}$. It may be tmagined that at $s=s_{a}$ there are no or very few electrons resonant with the wave, whereas at $s=s_{b}$ there are a substantial number of electrons, hopefuly with normalized parallel relocity $v_{1} / v_{\text {te }}=w \gg 1$, that are resonant with the wave. Thus, so satisfies the equation

$$
1=\int_{s_{b}}^{s_{a}} \alpha(s) d s=\int_{s_{b}}^{\infty} a(s) d s=\frac{\pi \omega_{p e}^{2}}{k_{s} c n_{o} \Delta v_{b}} \int_{s_{b}}^{\infty} n_{c} d s .
$$

The integral is perhaps more transparent in w-space where we write

$$
\frac{n_{r}}{n_{0}}=\frac{\Delta w}{\sqrt{2 \pi}} e^{-w^{2} / 2},
$$

and 


$$
\frac{d w}{d s}=\frac{1}{v_{\text {te }}} \frac{d}{d s}\left[\frac{\omega-\Omega_{e}(s)}{k_{\|}}\right]=\frac{\Omega_{e}}{k_{1} F_{\text {te }}} \text {. }
$$

so that Eq. (8) becomes

$$
1=\sqrt{\frac{\pi}{2}}\left(\frac{k_{\mathrm{H}}}{\mathrm{k}_{\mathrm{s}}}\right)\left(\frac{\omega \mathrm{pe}}{\Omega_{\mathrm{e}}}\right)\left(\frac{R}{c / \omega_{p e}}\right) \frac{\mathrm{e}^{-w^{2} / 2}}{w}
$$

where $w$ is the normalized resonant parallel velocity at $s=s_{b}$.

For fusion grade plasma, it is eas1ly seen from bq. (11) that $\geq 4$ is attalnable consistent with fuIl damping of the extraordinary wave. Further optimization (i.e., damping at higher w) can be obtained by minimizing $k_{s}$ for a given $k_{1}$. This corresponds to angling the wave not only in the toroidal direction, but also in the vertlcal alrection as 1 enters the plasma.

Note that because of the exponential dependence on $w$, nearly all the wave energy is absorbed in a narrow range $\Delta w$ in $w$. This corresponds to a narrow width $\Delta s$ in $s$. To estimite the damping width $\Delta s$, consider that a Maxwellian exponentiates in a width $\Delta w=1 / w$. Thus, making use of Eq. (10), we find that $\Delta s=k_{\|} R v t e^{\Delta w / \Omega_{e}}$ or in normalized paranteters

$$
\frac{\Delta \mathrm{g}}{\mathrm{a}}=\frac{0.1}{\mathrm{~T}_{10}^{1 / 2}}\left(\frac{\mathrm{R}}{3 \mathrm{a}}\right)
$$

where a is the wnor radius and $I_{p o}$ is the temperature normalized to 10 kev. In the regime $T_{10} \geq 1$, pertinent to reactors, it is seen that $\Delta s / a$ is indeed smal1. The heating and current generation profiles can, however, be much broader than is apparent at first glance. This is because $\Delta s$ on ${ }^{\prime}$ measures distonce paraliel to $b_{R}$. In fact, by vertically angling the wave 
( $k_{s}<<x_{1}$ ) not only is the current generated at higher $w$, but the deposition profile is broader since the wave damping now occurs over a longer length that intersects many magnetic surfaces.

In order to determine which is actually the best configuration for current generation, a fulI propagation study, such as has been conducted for the heating profiles, ${ }^{6}$ would have to be undertaken. Hote that for a given plasma (i.e., density and temperature profiles and dimensions) there is the opportunity to vary flve wave parameters: the frequpniy, whteh determines the vertical resonance surface; the polotdal angle at which the waveguide intersects the piasma periphery; the angles of injection, both in the toroldal direction and in the vertical direction; and, finally, the fifth parameter is the narrowness of the wave spectrum for a given power. The power is given roughly by the amount of current to be generated. The fifth parameter involved in the optimization dictates whether this power is to be concentrated in a narrow spectrum of $k_{1}$ or not.

III. CHECK OF THE LIMEAR THEORY

A formula was derived in Fef. 1 for the quantity $J / F_{d}$ for the case where the waves pugh electrons at velocities much exceeding the thermal velocity. This analygis represented a significant advance on the previous one-dimensional theory ${ }^{2}$ in that it distinguisheg the scatcering of electrons in pitch angle and energy. Furthermore, it treats the case of electron cyclotron damping which was not covered by the one-dimensional treatment. The important approximation made in Ref. 1 was the neglect of diffusion in the energy directfon. (Slowing down only was included in this direction.) The derivation itself is only valid when the speed of the resonant electrons far exceeds the electron thermal speed. We now seek to check the 
theoretical result of Ref. 1 by computing $\mathrm{J} / \mathrm{P}$, Erom a numerical solution of the two-dimensional Fokker-Planck equation.

The Fokker-Planck program used is the same as described in Refs. 4 and

7. That 1s, it solves

$$
\frac{\partial}{\partial \tau} f=\frac{\partial}{\partial \vec{u}} \vec{D}_{r f} \frac{\partial}{\partial \vec{u}} f+\left.\frac{\partial f}{\partial \tau}\right|_{\operatorname{coll}},
$$

where $\vec{D}_{r f}$ is the wave diffugion tensor (a function of $\vec{v}$ ) and the colligion tern $\partial f /\left.\partial t\right|_{\text {coll }}$ is calculated assuning flxed, constant temperature backgrounds of electrons and lons. As before, we adopt a normalization where $\tau=v_{0} t \quad\left[v_{0}=\log \Lambda \omega_{\mathrm{pe}}^{4} /\left(2 \pi \mathrm{n}_{0} \nabla_{t e}^{3}\right)\right], \quad t \vec{u}=\vec{v} / v_{t e}\left(v_{t e}^{2}=T_{e} / m\right)$. Normalized current and power dissipation are measured in units of en $\mathrm{v}_{\mathrm{V}}$ and $\nu_{0} n_{0} T_{e}$ respectively. In addition we define $x$ and $w$ to be the perpendicular and parallel (to $\vec{B}_{0}$ ) components of $\vec{t}$. The domain of integration is $u \leq 10$ with the condition that there be no flux of particles nornal to the boundary. Normally, we will only be concerned with cases where only the 获 (cyclotron damping) or the Ht (Lanliat iamping) component of $\bar{D}$ is nonzero.

$$
\begin{aligned}
& \text { With these normalizations } J / P_{d} \text { is predicted to be }{ }^{1} \\
& \frac{J}{P_{d}}=\frac{\hat{s} \cdot \nabla\left(w u^{3}\right)}{s \cdot \nabla u^{2}} \frac{4}{5+z_{i}} \text {. }
\end{aligned}
$$

where $\xi$ is a unit vector in the direction in which the wave pushes the electrons (i.e., $\vec{\xi}=\overrightarrow{\mathbf{x}}$ for cyclotron damping and $\vec{\xi}=\vec{t}$ for Landau damping) and $z_{1}$ is the ilon charge state. In practice ET. (13) should be integrated over the spectrum of waves. A further complication arlses if the waves are strong enough to alter the distribution function $f$ significantly because 
then $f$ must be determined before Eq. (13) can be applied. These difficulties rule out a detailed comparison of Eq. (13) with the numerical results obtained for the Landaw damping case. 4

Here we circumvent these difficulties by choosing $\bar{D}$ to be small and localized in veloctty space. Although the locallzation of $\vec{D}$ may be difficult to realize, this approach does allow us to check the physics embodied in Eq. (13). We take $D_{I f}$ to have a form which is zero except for $w_{1}<w<w_{2}$ and $x<1$ where it is equal to a constant $D$ multiplying either $\overrightarrow{x x}$ or $\vec{w}$. We take $w_{2}-w_{1}=1$ and $D=10^{-3}$, Figure 2 shows $J / P_{d}$ plotted as a function of $\left\langle w^{2}\right\rangle$ where tize average is computed with a Maxwellian weighting. There is excellent agreement between the numerical results (the symbols) and the analytical predictions (the lines). Interestingly, the theory and numerical results appear to agree fairly well even with w small for the cyclotron damping case but not for the Landau damping case. This is because the parallel input of momentun begins to be very substantial for low-phase veloctty waves ${ }^{7}$ and $J / P_{d}$ must begin to increase as 1/w. This effect is not present for the ECRH. Note, however, that these numericai calculations were performed assuming the background electrons to be nondrifting. If this constraint is relaxed, 7 the results for $J / P_{d}$ for $w_{1,2} \leq 1$ should be increased by a factor of about 2 .

\section{NONLINEAR RESULTS}

vormaliy we will be interested in cases where $D_{\text {If }}$ is large enough to pezturb $f$ significantly. To illustrate the type of behavior we might expect, we show in Fig. 3 plots of $f$ for $D+\infty$ and $w_{1}=4, w_{2}=5$ finc the cases of cyclotron and Landau damping. (As 1n the previous sect1on, we take Drf to be a constant $D$ for $w_{1}<w<w_{2}$. The perpendicular extent of: the waves was detentined only by the integration region, $u \leq 10.1$ 
A novel method was developed to treat the ease of $D \rightarrow \infty$. This is based on the observation that $\vec{s} \cdot \partial f / \partial \vec{v}$ must be zero. (Recall $\vec{s}$ is the direction in which the waves accelerate the particles.) This is achieved by replacing the diffusion operator by an averaging operator wiser the averaging is performed in stripg aligned with t.

Returning to Fig. 3, we first of all note that the perturbation to $E$ is much greater in the cyclotron dauping case. The reason for this is that the waves accelerate the particles so that they tend to stay in the resonant region. The waves are, therefore, more effective at acceierating the particles than waves which interact with partisles via the Landau resonance. The greater perturbation in"the cyclotron damping case means first that more current is generated ifor Fig. $3, J=3 \times 10^{-3}$ for cyclotron damping and $6 \times 10^{-4}$ for Landau dampingl. Since inuch of this current is carried by relatively collisionless particles with high perpendicular velocity, $J / P_{d}$ ig approximately twice its value in the low-D limit. (For $w_{q}$ $=4$ and $w_{2}=5, J / P_{d}=37^{\circ}$ for $D+\infty$ while $J / P_{d}=17$ for $D+0,1$ In fact, the cyclotron damped waves have overtaken the Landau damped waves which at low $D$ were more efficient in terma of $J / \mathrm{P}$. (For Landau damped haves with $w_{1}=4$ and $w_{2}=5, J / P_{d}=31$ for $D \rightarrow \infty$ and $J / P_{d}=26$ for $D \rightarrow 0.1$

The ease with which cyclotron damped waves can perturb $f$ has one interesting consequence, namely, that the power dissipated by the wave loes not necessarily saturate as $D$ is increased. ( $P_{\mathrm{d}}$ did saturate for the case shown in rig. 3 because of the effective cutoff on $D$ at $u=10$, such a saturation does occur with waves which are Landau damped and the resu:ts in the damping rate becoming zero as $D+\infty$. With cyclotron-damped waves, the behavior of the damplng rate as $D$ varies is a function of the $v_{1}$ dependence of D. In particular, even when $n$ is larye enough to graatly distort $E$ the damping rate may be fairly close to the linear damping rate. 
Figure 4 shows $J / P_{d}$ and $P_{d}$ as functions of $D$ for $w_{1}=4$ and $w_{2}=5$, As $D$ is increased, $J / P_{d}$ shows a steady rise while $P_{d} i s$ very nearly proportional to $D$ showling the constancy of the damping rate. This is so even though the distribution at the highest value of $\mathrm{n}$ given in Fig. 4, D = 0.25 is far from a Maxpeilian; see Fig. 5. For comparison, the Lower hybrid case is illustrated in Fig. 4 also. Nite the string gaturation of $P_{d}$.

A errisliary of the nearly linear behavior of $P_{d}$ with $D$ is that the damping of $*$ particular component of the wave spectrum is nut greatly affected by this neighboring components. This is illustrated in table I where the cases of $\left(w_{1}, w_{2}\right)=(4,5),(5,6)$, and $(4,6)$ with $D=0.1$ are compared. ive see that $J$ and $P_{d}$ for the $(4,6)$ case is given to within 108 by the sums of the $(4,5)$ and $(5,6)$ cases. On the other hand, the Aiscre $2-y$ with lower hybrid waves ts a factor ef about 2.

The results presented in this section need to be taken with sone caurion because when $D_{\text {rf }}$ is constait (as in these computations), there is a possibility of a runaway in the perpendicular direction since at high $v_{1}$ the collisions arn not able to hold the elentrons back effectively so that parti:-ies would be continuously accelerated in $v_{1}$ precluding the establishment of a steady state. The piesence of the numerical cutoff of the waves at $u=10$ would then dramatically alter the results. However, in practice the finite perpendicular wavelength of the waves causes $D$ to take a Bessel function dependence so thit $D_{\text {rf }} \sim 1 / v_{1}$ for hlgh $v_{1}$. (In the special casc of linear polarization, there is a cancellation which results in $\left.D_{\text {tf }}-1 /{ }^{3},\right)$ This decay of $D_{r f}$ is probably sufficiently fast to ensure the existence of a steady state. (We are assuming that at large p the effectiveness, if $D_{\text {rf }}$ is diluted by ine geometrical factor arising from the fact the fraction or the velocity space shell at u sccipied by the resonant 
region is proportional to $1 / u$. The effective $D_{r f}$ then decays as $1 / u^{2}$ which 1s at the same rate $s \mathrm{~s}$ the frictional term in the Fokker-planck equation. This allowg the establishment of a stesdy grate in which $E$ decays exponentially with u.)

Now the $1 / v_{1}$ dependence takes over at $v_{1} \sim g_{e} / k_{1} \sim c$. For typical electron temperatures $(\sim 10 \mathrm{keV})$ this would be $x \sim 10$. If we solve for $\mathrm{E}$ wth a boundary at $u=10$, then the arror entailed by introducing the boundary will be small if $f$ at the boundary is small since we know $f$ in fact decays exponentially beyond the boundary. Because the decay rate depends on $D_{\text {ff }}$ we must also restrict $D_{\text {If }}$ from teing too large. In order to determine the behavicr of the particles at larger values of $D_{r f}$ where the large $v_{1}$ behavior of $D_{r t}$ is important we must include relativistic effects becauge the velocities of these particles are close to that of light. This is addressed in the next section.

\section{RELATIVISTIC EFFECTS}

In this section we outline how various relativistic effocs may play a role. These effects, which are pertinent to fusion grade plasmas, are all rather weak in the lower tenperture reactors $\left(T_{10}<2\right)$, but become increasingly important at the higher temperatures that are sharacteristic of more advanced reactors.

The wost important relativistic effect relates to the efficiency of current generation. As described in Ref. 3, this efflciency is somewhat reduced for mildly relativistic electrons, but approaches zero for very relatfistic electrons. In view of this inefficiency of relativistic electrons, thio effect supercedes the additive deleterious effect of power logs through synchrotron radiation of very fast electrons. 
There are other relativistic effects that impinge on the wave-particle interaction itself. One effect is the decrease in $D_{r f}$ due to finite gyroradius effects, which is an effect avallable nonrelativistically too. We turn here, however, to more cundamentally relativistic effects. In o.u analysis, we assumed that electrong were pushed by the wave only in $v_{1}$ space where they remained in resonance with the wave. The result of this would be that, in the absence of collisional effects, there would be complete flattening in $v_{1}$ space for the resonant electrons, $1.4 .1+0$. Relativistic effects change this plature in two way3. First of all, as the electron perpendicular energy increases, so does its felativistic masa, which means that it car fall out of resonance. To be specific, the resonance conditiol, is now

$$
v_{1}=\left(\nu-\gamma_{e}(\gamma) / k_{1},\right.
$$

where $\gamma=\left(1-v^{2} / c^{2}\right)^{-1 / 2}$ and $\partial_{e}$ is the cycictron Ereyuency of nonrelativistic electrons. The result of the electron falline sut of resonance is that the flattening in $v_{\perp}$ cannot contfoue and f will not vanish anywhere in the colitsionless limit.

The second ielativistic effect inpinging on the flattening of felates to the direction in which electrong are diffused. This diffusion path nay be thought of as that sector of velocity space where $f$ is induced to be

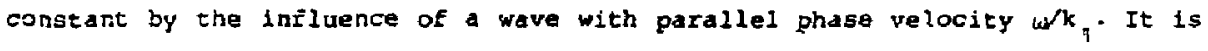
given by

$$
E=p_{1}+V_{k}=\text { constant }
$$


which is the relativistic statement of congervation of eneroy and parallel momentum. The effect of this is again to push electrons not purely in the perpendicular direction and and hence to push them out of the reyonance with tixe wave. Now, $f$ becomes normallzatle in the collisionless limit. To appreciate 5q. (14), it is helpful to plcture these diffusion. patis. In the nonrelativistic 11mit, Eq - ( 14$)$ describes the familiar concentric circles in veloeity space about the goint $\left(v_{1}=0, v_{1}-\omega / k_{1}\right)$. Consider, however, Fis, 6(a) where diffusion paths cue to a wave at $\omega / K_{1}=0.5, c$ are depicted. The faniliar circles are now seen to be somewhat distorted in order to assure that the particle velocity remain less than c. In Flg. 6(b) we ghow diffusion paths due to a superluminous wave with $\omega / k_{i}=2 \mathrm{c}$. Here, the topology of the paths are seen to have cha!nged from ellipsoidal to hyserboloidal surfaces. ithe topology change is characteristic of supierluminous waves and occurs in momentic space too. In Fig. 6(c) we show the iiffusion paths in momentum эpace for $\omega / \mathrm{k},=1.1 \mathrm{c}$.

We now turn to relativistic effects that impinge upon technological considerations. Erom an engineering standpoint, it woudd be most preferred to use a wave of the lowest possible frequency and to inject it from the low field side of the tokamak.

consider firgt the launching of the extraordinary plasma wave which must be done from the high-fisli side if ccadition $k_{1} v_{1}=w-\Omega_{e} / r$ may be witten as

$$
\left|\frac{\omega}{\Omega_{\mathrm{e}} / \gamma}\right|=\left(1+\frac{v_{1}}{\omega / k_{1}}\right)^{-1}
$$


and two implications with respect to minimizing w became apparent. Note that $w / k_{f} 2 \mathrm{c} ;$ however, at reactor grade temperatures, $\nabla$ may approach $c$. Thus, we may bound

$$
\frac{1}{2}<\frac{w}{\Omega_{e} / \gamma}<1 .
$$

and the lower (preferable) limit is accessible only for rearly parallel Injection $\left(k, \gg, k_{1}\right)$ and bsorption by relativistic elertrons. This limit is preferred because it minimizes the frequency of the power source.

The second point to be made about Eq. (15) is that for relativfstic electrons $Y$ becomes large, which has the effect of requiring a smaller Erequency powti source.

For the ordinary wave, which is launched frem the low flela side, similar considerations are pertinent. Hert we have

$$
\left|\frac{w}{\Omega_{e} / \gamma}\right|=\left(1-\frac{k_{1}^{y}}{w}\right)^{-1},
$$

and, for this waye $k_{n}$ small toplies $\omega$ smallar and $\omega>\Omega_{e} / \gamma$ ls always true.

\section{I. CONCIUSIONS}

We have examined varioug aspects of current drive by electron cyclotron waves. Although the ourrent drive mechanism is intrinsically less efficient than for surrent drive by lower hybrid waves, the greater flexibility of positioning the wave spectrum and the ho:linear enhancement of $J / P_{d} g$ iven in section IV offset this disadrantage.

The most interesting result of the numerical studies of the nonlinear problem is that the damping rate of the wave is nearly independent of the 
power level. The wak dependence that there is is masked by the exponential Eactor $\exp \left(-w^{2} / 2\right)$ entering the formula for the power dissipated. Inis means that ray-tracing codes my safely uge linear damping theory. Nonlinear effects became important in estimating the current drive efficiency, where the nonlinearity can eriance $J / P_{d}$ by up to a factor of about 2 .

In order for the current to be generated in a single direction, we require that all the power be absorbed before the cyciotron Iayer. In Section II it was shown that this wag eagliy achleved wh the extraordinary wave In i reactor. An additional margin of safety is provided, however, by positioning the source close to the top or bottom of the machine ibut st111 in the high-field side of the cyclotran layer) since the group velocity. normal to the cyclatron layer is, thereby, reduced. such an arranagement also allows easier access for the waveguides.

We have presented here only the numerical results for the extraordinary wave for which $D$ is independent of $v_{\perp}$ for low $v_{1}$. For the ordinary wave the polarization of the wave is sueh that the electric field vector is rotating in the opposite direction to that of the electrons. The diffusion coefflcient $D$ then behaves as $v_{1}^{4}$ for low $y_{1}$. This is more difficult to model numerically because of the greater importance of an accurate treatment CI the behavior of large $v_{1}$ particles. we expect that for a given $w_{1}$ and $w_{2}$ the efficiency of current generation will be greater because more of the current is carrled by collisionjess high-velocity particles. However, the damping rate 13 also weaker so that more current will be generated at lower values of $w_{1}$.

Several phenomena influence the diffusion of electrong at high $v_{\perp}$. Finite wavelength effects cauge the diffusion coefficient to oscillate with 

relativistic effucts which change both the diffusion paths and the resonant region. Relativistic eftects also allow the use of frequencles substantially below the gyclotro frequency. Inis will be important in hot second-generation reactors.

\section{ACKNOWLEDGENTS}

The authors would like to thank E. Ot for useful discussions. This work was suported by the United states Department of Energy under contract number $\mathrm{DE}-\mathrm{ACO} 2-76-\mathrm{CHO} 03073$. 


\section{REFERENCES}

[1] EISCH, 4 , J. and BOOZER, A. H., Phys. Rev. Lett. 45, 720 (1980).

(2) F.SCH, N. J., Phys. Rev. Lett. 41, 873 (1978) and 42, 410 (1979).

[3] FISCH N. J., Penceton Plasma Fhysics Laboratory Report PPPL-1739 (1981).

[4] KRRNEY, C. F. F. and FISCH, N. J., Phys. Fluids 22, $1817\{1979\}$.

[5] KENNEL, C. F, and ENGELMANN, E., Phys. Flulds 9, 2377 \{1966\}.

(6) OTT, E., HUI, B., and CHU, K. R., Phys. Fluids 23, 1031 (1980).

[7] FISCH, N, J, and KARNEY, C. F. F., Phys, Flulds 24, 27 (1981). 
TABLE I.

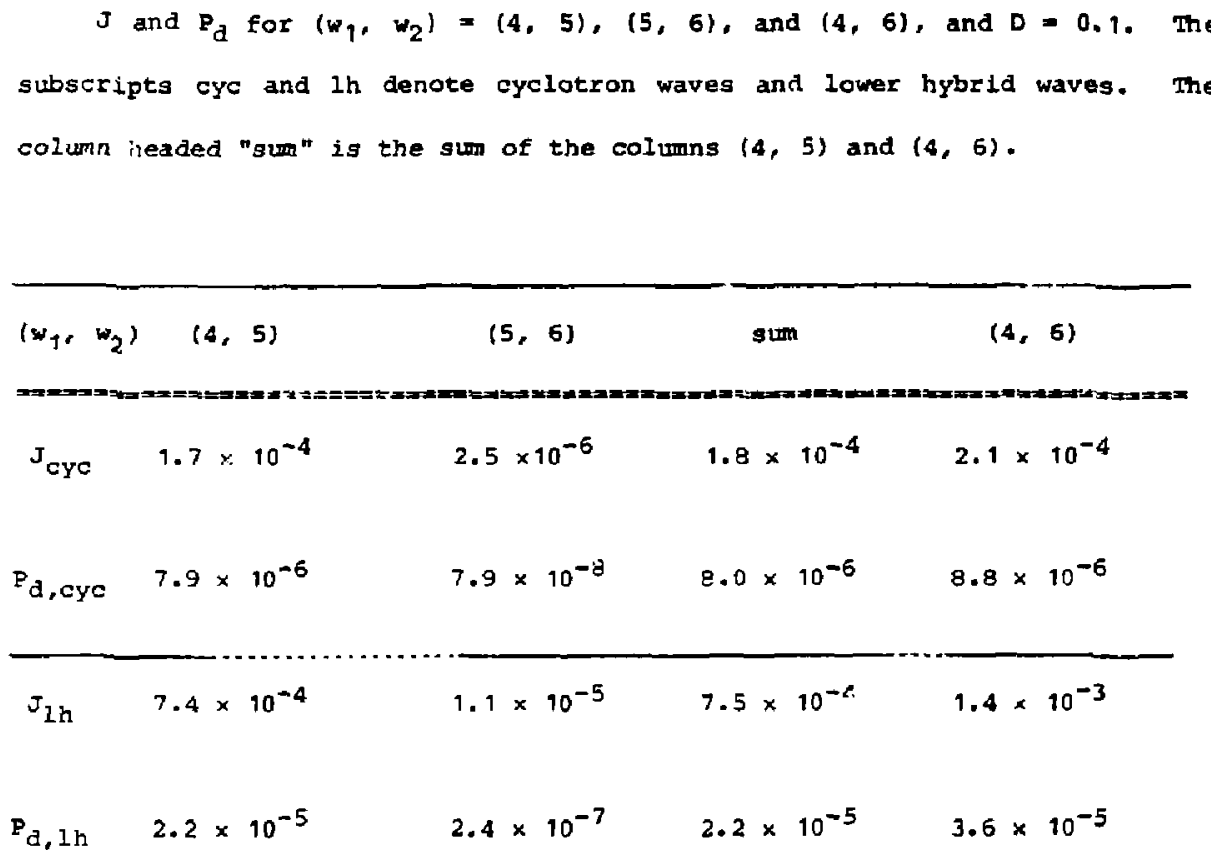




\section{FIGURE CAPTIONS}

Fig. 1. Comparion of electron cyclotron and lower hybrid methods of current drive. The wave spectrum and distribution function for the lower hybrid (a) and the electron cyclotron (b) methods of eurrent drive.

Fig. 2. $J / P_{d}$ for swall $D$ as a Eunction of $\langle w\rangle$ where $D=10^{-3}, w_{2}-w_{1}=$ 1. The waves exist caly for $x<1$. The open circles denote cyclotron damping and closed circles Lanaau damping. The lines show the theoretical predictions of Eq. (13).

Fig. 3. The steady-state distribution functions for $D \rightarrow \infty$ with $w_{1}=4$, and $w_{2}=5$. Figures (a) and (b) show the cases of electron cyclotron waves and lower hybrid waves, respectively.

Fig. 4. $J / P_{d}$ (a) and $P_{d}(b)$ as functions of $D$ for $w_{1}=4$ and $w_{2}=5$. The open circles denote cyclotron damping and the closed circles Landau damping.

Fig. 5. Steady-state distribution for $D=0.25, w_{1}=4$, and $w_{2}=5$ (cyclotron damping).

Fig. 6. Diffusion paths for particles in waves. Velocity space with (a) $\omega / k_{1}=0.6 \mathrm{c},(\mathrm{b}) t / \mathrm{k}_{1}=2 \mathrm{c},(\mathrm{c})$ momentum space with $w / k_{1}=1.1 \mathrm{c}$. 

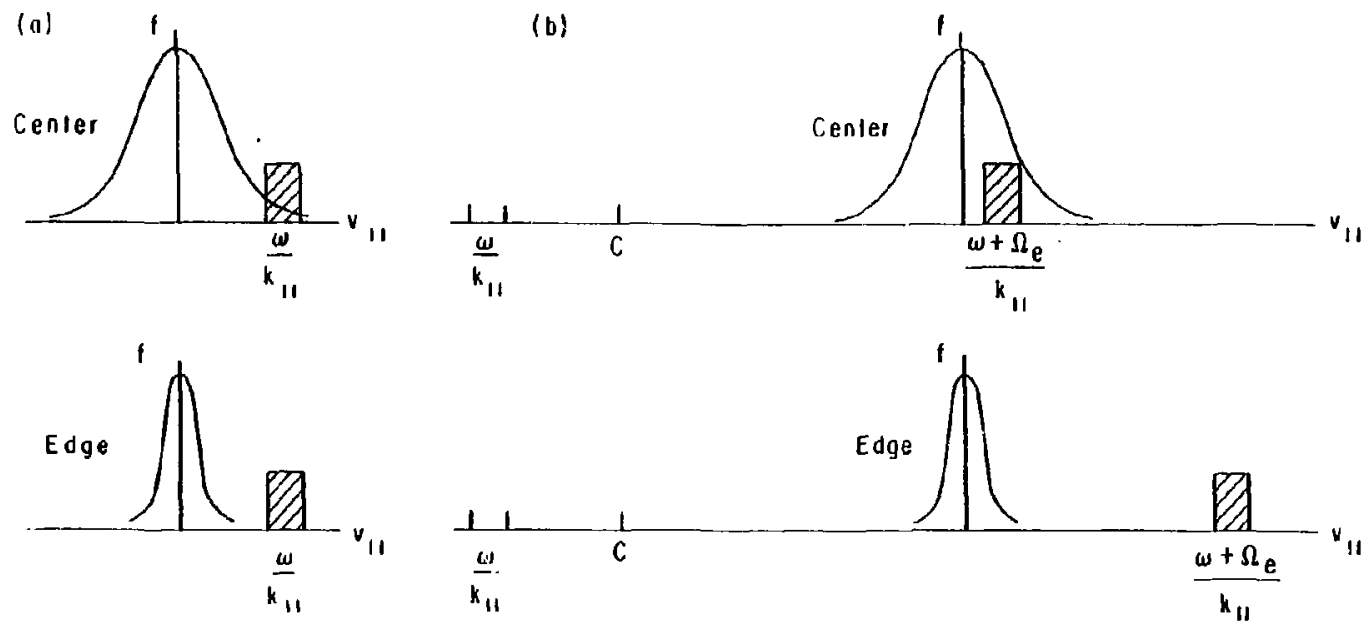

Pig I Comparison of electron vyclotron and lower hybrid methods of currut drive. The wive spectium and distrjbution function for the lower liytejd (a) and the eledtron cyclotron (h) inetlods of cur rent drive. 
* 8150122

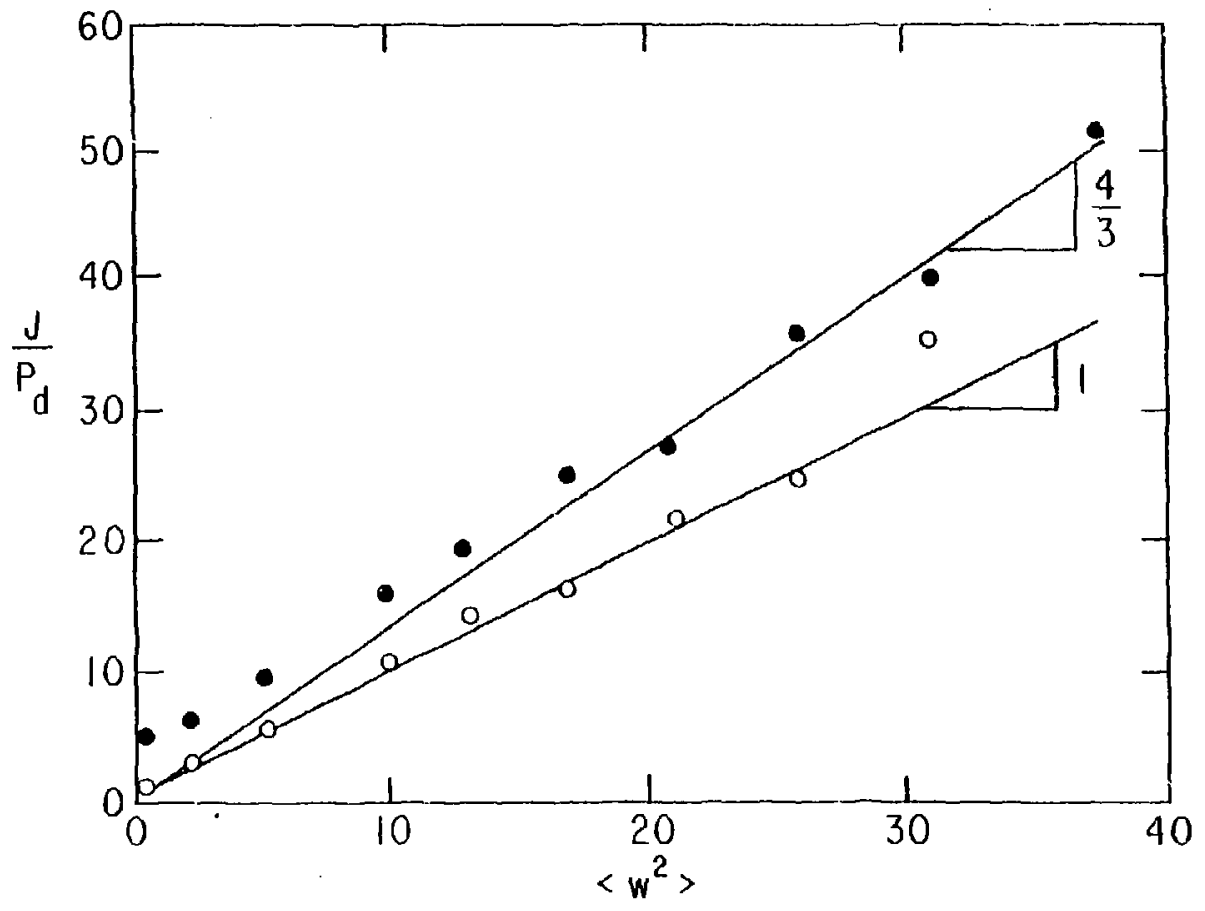

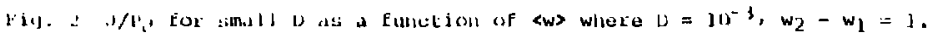
Tlue waves ex itit. mily for $x<1$. Ithe open circles denote cyclution

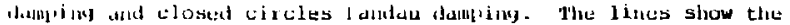
Lheorelical predictions of Éd. (1J). 

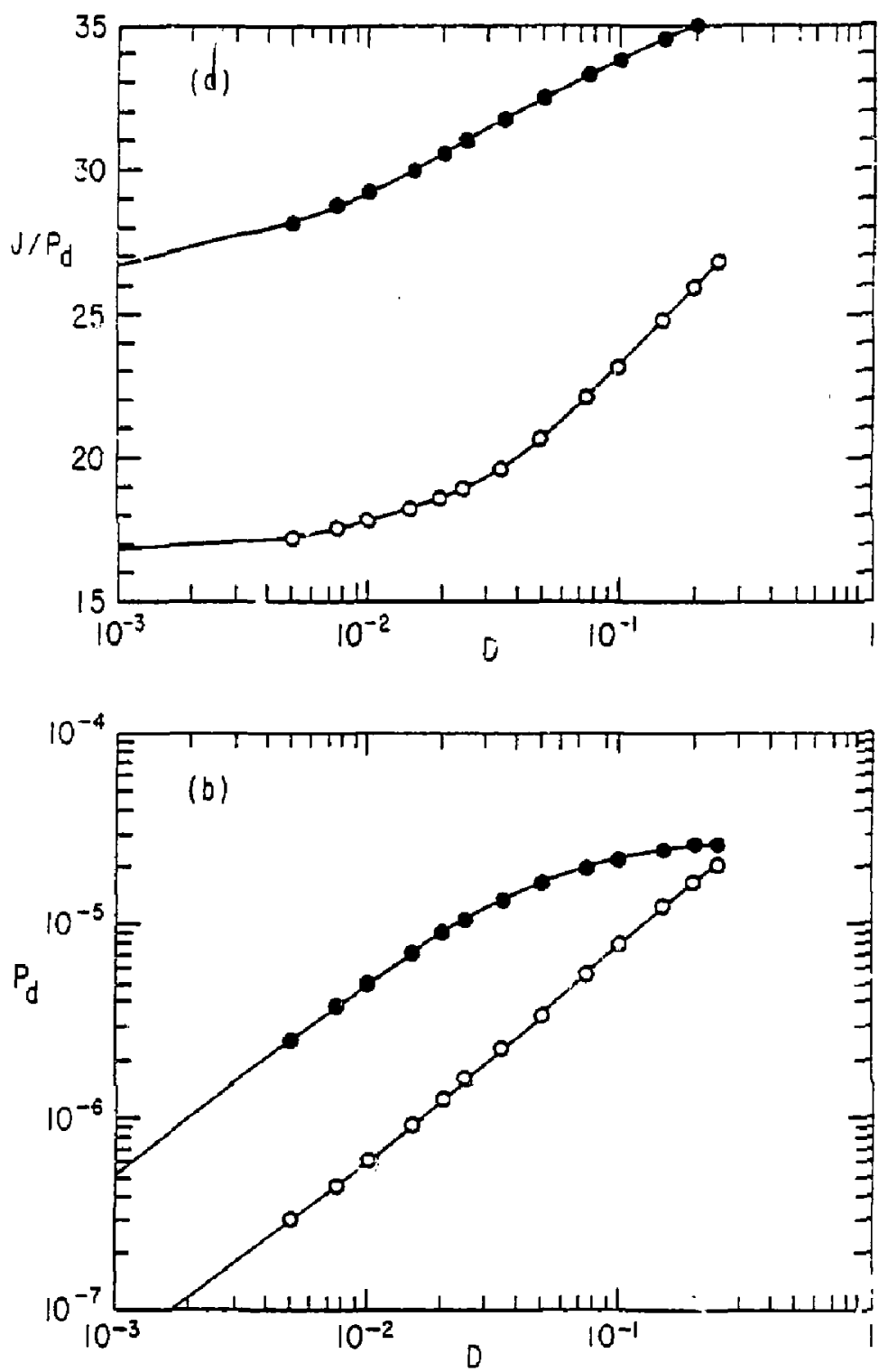

Fig. $4 \quad J / \Omega_{\mathrm{d}}(a)$ and $z\left(\mathrm{~b}\right.$ (b) as Eunctions of $\mathrm{D}$ for $w_{1}=4$ and $\mathrm{w}_{2}=5$. The open circies denote cyolotzon damping and tile closed circles Landau dampiag. 
\# 81т0119

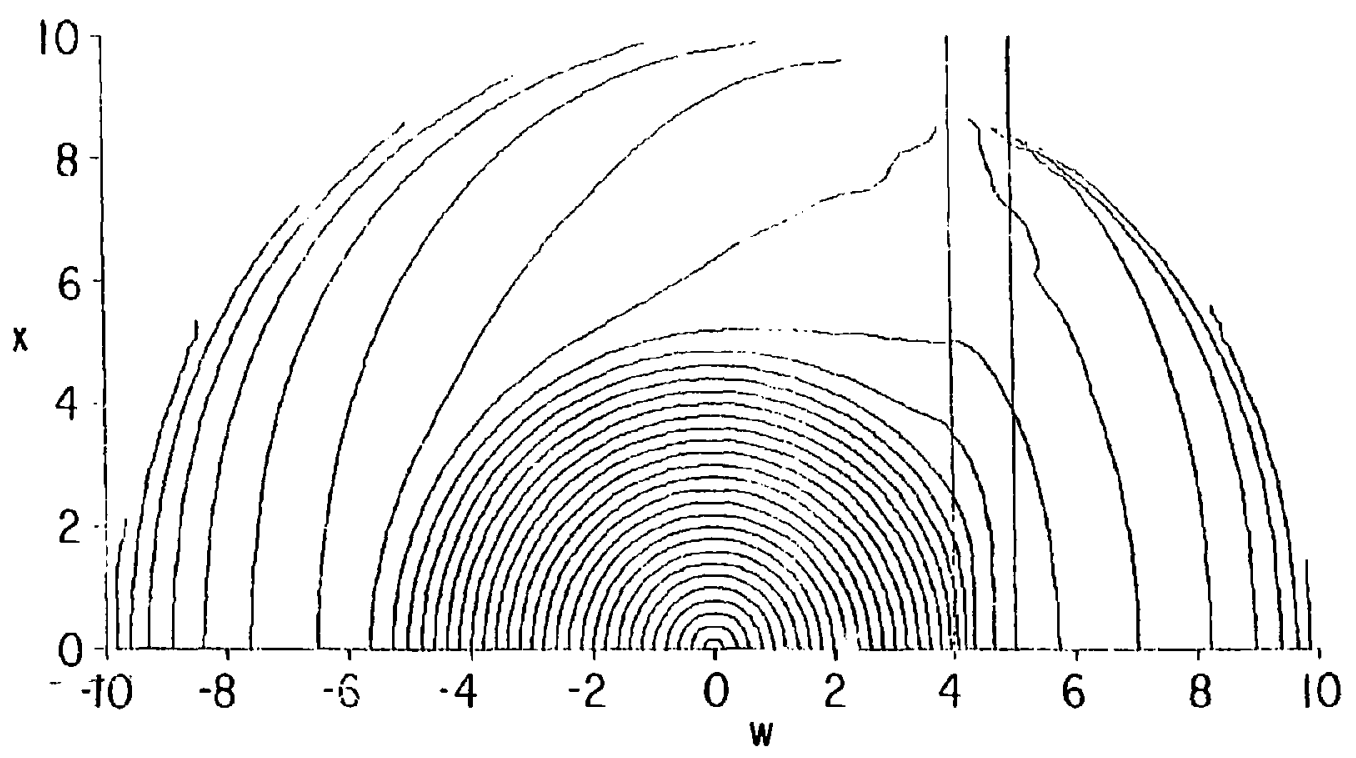

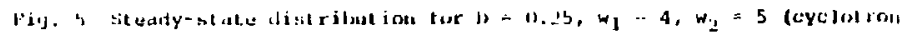
(dolninini). 

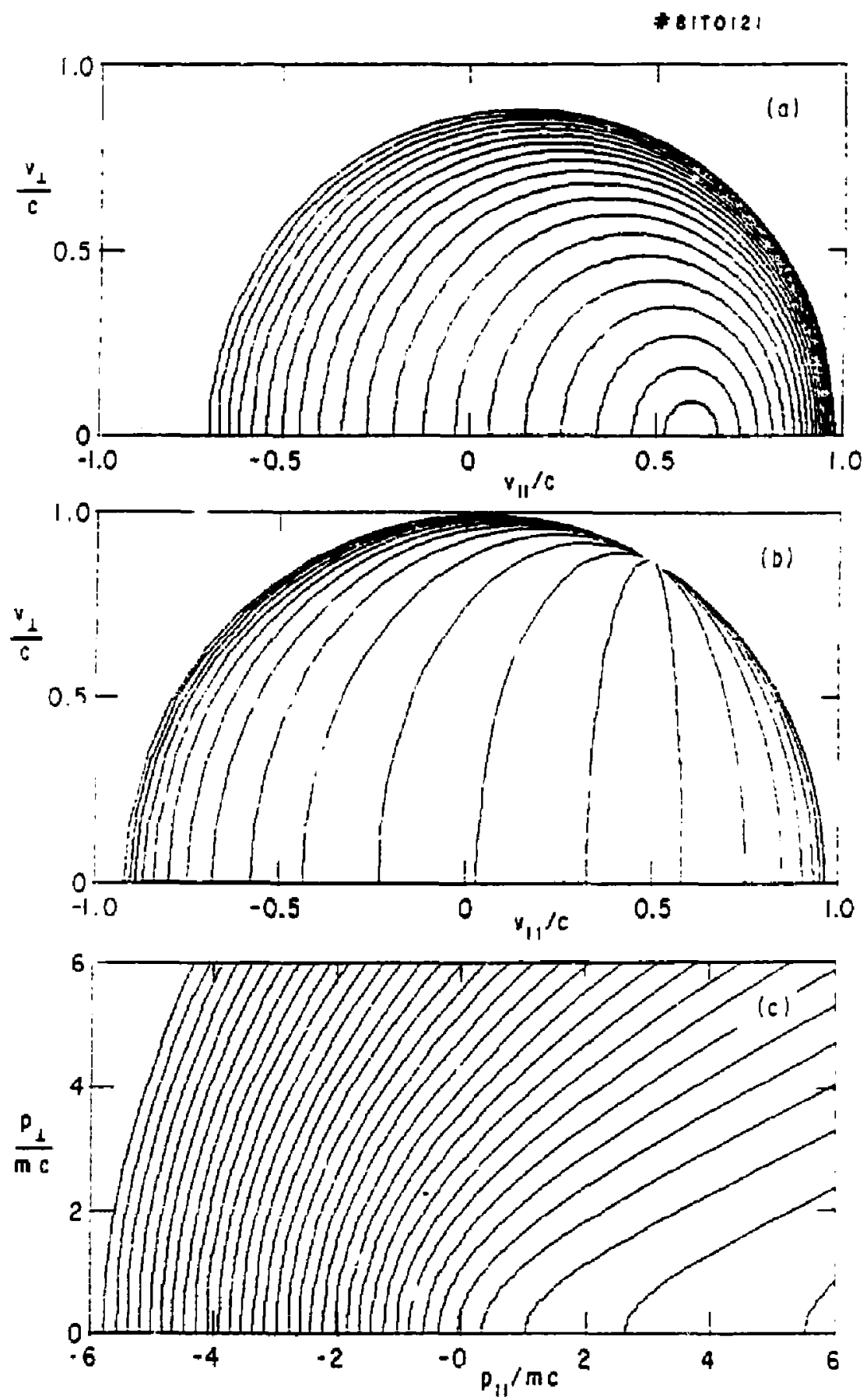

Ėg. 5 Diffusion paths for particles in waves. Velocicy space wit:? (a) $-/ k=0.0 c$, (b) $u / k_{j}=2 c$, (c) monentum space witi $\perp / k_{i 1}=1.1 c$ 
ALL CA TECORIES

R. Askew, Auburn Uni versi ty, Alabama

5. T. Wv, Unv. of Alabana

Geopliysical Instirute, L'nuv. ol Alaska

G.L. Johnston, Sonoma State Univ, Caj jornia

H. H. Kuehl, Uni v. of S. California

Institute for Energy Studies, St and ord Universisy

H. D. Canpbell, University of F lorida

N. L. Olesan, University of South Florida

W. M. Stacey, Georgia Institute of Technolots

Denjamin Ha, lowa State University

Magne Kristionsen, Texas Tech. Uri versity

W. L. Wiese. Narl Bureau of Standar $\$$, Wash., D.C.

Australian National Uni versity, Canberra

C.N. Watson-Munro, Univ. of Sydney, Australia

F. Caf, Inst. ior Theo. Physics, Austria

Dr M. Heinder, Institute for Theoretical Physics

Tectuical University of Graz

Ecole Royale stilizaire, Bruxelles, Beigium

D. Palumbo, C. European Comm-B-1049-Brussed s

P.H. Sakanaka, Instituto de Fisica, Campinas, Braz.]

M.P. Bachynsk, MPB Tech, Ste. Ame de Bellevue,

Quebec, Canada

C. R. James, University of Alberta, Canada

T.W. Johnston, INRS-Energie, Vareenes, Quebec

H. M. Skarsgard, Univ. of Sasteaichewan, Canada

Insi. of Physics, Academia Siruca, Pef ng.

People's Republic of China

Inst. of Plasma Physic, Hefei,

Anhwel Pronna. People's Republic of Chn

Libr ary, T sing Hua IJniv., Pekng, People's

Reoublic of China

Thengwul.. Southwestern Inst. of Phys, Leshan,

Sichuan Province. People's Republic of China

Libearıan, Culham $L$ aboratory, Abingdon. Engl and

A.M. Dupas Library, C.E.V.-G, Grenoble, France

Central Res. Inst. for Physic, Hungary

S. R. Sharma, Univ, of R jyast han, JAIPLR -4, India

$R$. Shingal. Heer ut College, India

A K. Siundaram, Phys. Res. Lab. India

Bıbljoreca, Frascatı, ltaly

Biblioteca, Miluno, It aly

G. Rostagni, ij nu v. Di Pado va, Padova, Italy

Preprint Library, Inst. de Fisica, Pisa, It aly

Librar y, Plasma Physics Lab. Golasho, Uj, Japan

5. Wori, Jaoan A tomic Energy R es. Inst , Tokaj-Wura

Research information Center, Nagoya Unu $v_{7}$ Japan

5. Shuoda, Tokyo 'nst. of Tech., Japan

Inst. of Space \& Aero. Sa. IIniv. of Tolyo

T. Uchda, Univ. of Tokyo, Japan

H. Y an ato, Tos hiba R. \& D. Center, Japan

M. Yoshikawa, JAERI, Tokai Res. Est, Japan

Dr. Tsuneo Nalakita, Joshiba Corporation,

Kawasaki-Ku Kawasaij, 210 Japan

N. Yayima, K yushu U. Iniv, Japan

R. England, Univ. Nacional Auro-numa de Mexico

B. 5. Luley, Univ. of Waikato, New Zeal and

5. A. Moss, Saab Urivas Norge. Norway

J.A.C. Cabral, Univ. de Lisboa, Port ugal

O. Petrus, AL.I. CUzA Univ, Romania

J. de Villiers, Atomic Energy Bd, South A frica

A. Maurech, Comisaria De La Energy y Recoursos Miner ales, Spain

Library, Royal lrstitute of Tectmology, Sweden

Cen. de Res. En Phys Des Plasmas, Switzertand

Librarian, Fon-Institut Vur Plasma-Fysica,

The Netherl ands
Biblior hek, Stutigart, West Germany

F.D. Buhler, Univ, of Stungart, West Germany

Max-Planck-inst . fur Plasmaphysik, W. Germany

Nucl. Res. Estab, Julich, Weat Germany

K. Schinder, Inst. Fur theo. Physik, W. Germany

\section{EXPERIMENTAI}

THEORETICAL

M. H. Brennan, Flinders Univ, Australia

H. Barnard, Univ. of British Columbia, Canzda

5. Soreeni vasan, Univ, of Calgary, Canada

J. R adet, C.E.N.-Q.P, Font enay-aux-Roses, France

Prot. Schatzman, Obser vatoire de Nice, France

5. C. Sharma, Univ. of Cape Coast, Grana

R. N. Aiyer, Laser 5ection, India

B. Buti, Phusical Res. Lab., India

L. K. Chavda, S. Gujarat Univ., India

I.M. Las Das, Banaras Hindu Liniv., India

S. Cuperman, Tel A viv Univ, lsrael

E Greenspan, Nuc. Res. Cent $\sigma$, Jsrael

P. R coerau, Israel Inst. of Tech 4 Israel

Int'l. Center Ior Theo. Physics, Jrieste, Italy

I. Kawakami, Nihon University, Japan

T. Nakayama, Ritouneikan Uny., Japan

5. Nagao, Tohoky Univ, Japan

J.]. Saka1, Toyama Univ., Japan

S. Tjotra, Univ. I Bergen, Norway

M.A. Hellber g, Univ. of Natal, Sour A A frica

H. Whelmson, Chalmers Univ. of Tech., 5 weden

A stro. Inst ., Sonnenbor ght Ots,

The Netherlands

T. J. Boyd, Unv. College of North W ales

K. Hubnet, Univ. Heidelber $8, W$, Germany

H. J. Kaeppeler, lins. of 5 cunt gart,

West Germany

K. H. Spats thek, Uruv. Essen, West Germany

\section{EXPERIMENTAL \\ ENGINEERING}

B. Grek, Unu, du Quebec, Canada

P. Lukac, Komenskeho Univ., Cuechoslovakua

G. Horitoshi. Nat'l Lab for High Energy Physics, Tsukuba-Gun, Japan

\section{EXPERIMENTAL}

F. J. Paoloni, Uiuv, of Wollongong, Australia

1. Kistemaket, Fom Inst, Ior Atomic d Molec. Physics, The Netherlands

\section{THEORETICAL}

F. Verthest, Irst. Vor Theo. Mech., Belgium

J. Teichmam, Univ. of Mon'real, Canada

T. Kahan, Univ. Paris VII, France

R. K. Chhajlani, India

5. K. Trehan, Panjab Unu $V_{y}$ India

T. Namikawa, Osaka City Unir, Japan

H. Nar uni, Unv, of Hiroshima, Japan

Korea A tomir Energy Res. Inst., Korea

E. T. Karlson, Uppsala U niv $v_{\eta}$ Jweden

$L$ Stenllo, Uhiv, of UAIEA, Sweden

J. R. Soral, New Univ., United Kingdom 